\title{
Effect of Acupuncture on Premature Ovarian Failure: A Pilot Study
}

\author{
Yingru Chen, ${ }^{1}$ Yigong Fang, ${ }^{1}$ Jinsheng Yang, ${ }^{1}$ Fei Wang, ${ }^{2}$ Yingying Wang, ${ }^{1}$ and Li Yang ${ }^{1}$ \\ ${ }^{1}$ Institute of Acupuncture and Moxibustion of China Academy of Chinese Medical Sciences, \\ No. 16 Dong Zhi Men Nei Nan Xiao Jie, Dongcheng District, Beijing 100700, China \\ ${ }^{2}$ Institute of Acupuncture and Moxibustion of the Hospital Affiliated to Tianjin University of Traditional Chinese Medicine, \\ No. 314 An Shan Xi Dao, Nankai District, Tianjin, 300193, China
}

Correspondence should be addressed to Yigong Fang; fangyigong@163.com

Received 31 October 2013; Accepted 5 February 2014; Published 10 March 2014

Academic Editor: Lixing Lao

Copyright (C) 2014 Yingru Chen et al. This is an open access article distributed under the Creative Commons Attribution License, which permits unrestricted use, distribution, and reproduction in any medium, provided the original work is properly cited.

To investigate the effect of acupuncture on women with premature ovarian failure (POF), prospective consecutive case series study was applied. 31 women with POF were included; all patients were treated with acupuncture once every other day, three times a week for three months. Acupoints, GV 20, GV 24, GB 13, CV 3, CV 4, BL 23, BL 32, ST 25, ST 28, ST 29, ST 36, SP 6, KI 3, and LR 3, were selected. Serums FSH, $\mathrm{E}_{2}$, and LH level, Self-Rating Anxiety Scale (SAS), and Kupperman score were measured at baseline and at the end of treatment; the menstrual cycle was recorded during one-month follow-up. Compared with baseline, patients' serums FSH and $\mathrm{LH}$ were decreased, $\mathrm{E}_{2}$ was increased, and SAS score and Kupperman score were decreased. Four patients resumed menstrual cycle after treatment and two resumed during follow-up. No serious adverse events were found in all patients. The results indicate that acupuncture may decrease serums FSH and LH level, raise serum $\mathrm{E}_{2}$ level, relieve anxiety, reduce mental stress, and improve the menopausal symptoms.

\section{Introduction}

Premature ovarian failure (POF) is a common cause of infertility in women and is characterized by amenorrhea before the age of 40 . Menopause before the age of 40 is considered to be premature and is nonphysiological disorder. POF, also known as premature menopause or premature ovarian insufficiency, is defined by the presence of menopausal-level serum folliclestimulating hormone (FSH) in women younger than 40 years [1-3]; the mean $( \pm \mathrm{SD})$ age of natural menopause is $50 \pm 4$ years [4]. This syndrome is associated with the symptoms and metabolic effects of sex steroid deficiency, as well as the emotional sequelae experienced by couples who have difficulty in conceiving a pregnancy. POF is a disorder affecting approximately $1 \%$ of women $<40$ years, $1 / 1,000$ women by the age of 30 , and 1/10,000 women by the age of 20 .

Women with POF have been reported to have diminished general and sexual well-being, are less satisfied with their sexual lives, have increased risk for low bone density, earlier onset osteoporosis and fractures, impaired endothelial function, earlier onset of coronary heart disease, and increased cardiovascular mortality and total mortality, and have more anxiety, depression, somatization, sensitivity, hostility, and psychological distress than normal women [5-7].

POF was not nominally recorded in Traditional Chinese Medicine (TCM). However, according to TCM theory, POF can be pertained to amenorrhea according to the clinical manifestations. As early as in 1237 A.D., the first book about gynaecology and obstetrics of Chinese Medicine, The Complete Book of Effective Prescriptions for Diseases of Women, said that acupuncture and Chinese Herbal Medicine usually led to satisfying symptom relieving effects in treating some gynaecology disorders, such as endometriosis, infertility, amenorrhea, and menopausal syndrome [8]. In recent studies, acupuncture has been found effective in reducing hot flashes of bilaterally ovariectomized patients [9] and women undergoing perimenopause and menopause [10-12] and significantly improving serum estradiol $\left(\mathrm{E}_{2}\right)$ level and 
TABle 1: Acupoints used in this study.

\begin{tabular}{ll}
\hline Prescription & Acupoints \\
\hline \multirow{2}{*}{ Prescription 1 } & CV 3 (Zhongji), CV 4 (Guanyuan), ST 25 (Tianshu), ST 28 (Shuidao), ST 29 (Guilai), and LR 3 (Taichong) \\
& GV 20 (Baihui), GV 24 (Shenting), GB 13 (Benshen), ST 36 (Zusanli), SP 6 (Sanyinjiao), and KI 3 (Taixi) \\
\hline \multirow{2}{*}{ Prescription 2 } & BL 23 (Shenshu), BL 32 (Ciliao) \\
& GV 20 (Baihui), GV 24 (Shenting), GB 13 (Benshen), ST 36 (Zusanli), SP 6 (Sanyinjiao), and KI 3 (Taixi) \\
\hline
\end{tabular}

Kupperman scores of perimenopause patients [13]. Acupuncture also has been proved to be effective in improving pregnancy outcomes in women undergoing in vitro fertilization (IVF) from summarized data of several previously published meta-analysis and lots of clinical trials [14, 15]. Although the therapeutic mechanisms of acupuncture in the abovementioned disorders are not yet to be fully understood, a plausible hypothesis is that acupuncture may influence related hormone levels.

However, there is less evidence on the effectiveness of acupuncture for patients with POF. This study, therefore, was designed to assess the feasibility and safety of acupuncture for POF patients on the basis of TCM theory, which includes local and distal acupoints to reinforce liver and kidney, to regulate Qi and Blood, and to adjust mental activity.

\section{Materials and Methods}

2.1. Study Design. This was a prospective consecutive case series study performed at the Hospital of Acupuncture and Moxibustion of China Academy of Chinese Medical Sciences. Participants received 3 sessions of acupuncture treatment, delivered 3 times a week for 3 months. The data from patients include the serums FSH, $\mathrm{E}_{2}$, and luteinizing hormone (LH) level, Self-Rating Anxiety Scale (SAS) [16], and Kupperman score [17], (The SAS and Kupperman score have been validated and widely used in Chinese population $[18,19]$.) The menstrual cycles were recorded during one-month followup. Adverse events were tracked for 3 months from initial acceptance of acupuncture treatment to the end of treatment.

The institute ethics committee approved this treatment protocol for women with POF (Institute of Acupuncture and Moxibustion Beijing (ethics) approval number: 20111117), and all patients signed informed consent before study participation. Acupuncture was performed by one therapist with more than 20 years of experience and Chinese medicine practitioner license from the Ministry of Health of the People's Republic of China. Data coordination, monitoring, analysis, and source verification were done by the researchers who did not take part in the clinical treatment. Figure 1 shows the study design.

2.2. Study Participants. Present study recruited patients through the following ways: (1) publish recruiting advertisement on the website of the Hospital of Acupuncture and Moxibustion of China Academy of Chinese Medical Sciences; (2) publish recruiting notification on the largest Chinese doctor information website; (3) place recruiting advertisement in registration hall of our hospital. Informed consent was obtained before participant enrollment according to a clinical trial protocol. Recruitment was performed from December 2011 to January 2013, and 31 cases of POF were recruited.

Inclusion criteria were as follows: (1) the patients meet the POF diagnosis criteria, mainly with amenorrhea for four months or longer and FSH above $40 \mathrm{IU} / \mathrm{L}$ as detected on at least two occasions with at least one month apart [20-22]; (2) the patients' age is from 18 to 40; (3) before treatment, all patients had gone through one-month baseline evaluation period during which they stopped all medications influencing reproductive hormones; (4) patients were advised and agreed not to use these medications during study. Exclusion criteria were as follows: (1) bilateral oophorectomy, gonadal dysgenesis, and chromosomal abnormalities; (2) ovarian failure caused by radiotherapy and/or chemotherapy of cancer; (3) autoimmune diseases and/or receiving hormones or immunosuppressant drugs; (4) serious primary diseases of cardiovascular, liver, kidney, and hematopoietic system; (5) having no desire to participate in the research.

\subsection{Interventions}

Acupuncture Needles. Sterile, silver-handle, prepacked needles (HanYi single-use acupuncture needle, made in Tianjin HuaHong Medical Co., Ltd.) without guide tubes (size $0.25 \mathrm{~mm} \times 25 \mathrm{~mm}, 0.25 \mathrm{~mm} \times 40 \mathrm{~mm}$, and $0.30 \mathrm{~mm} \times$ $75 \mathrm{~mm})$.

All acupoints were selected and localized according to WHO Standardized Acupuncture Point Location [23] (Table 1). Needles of the size of $0.25 \mathrm{~mm} \times 25 \mathrm{~mm}$ were inserted horizontally into GV 20 (Baihui), GV 24 (Shenting), and the bilateral GB 13 (Benshen) with a depth of $20 \mathrm{~mm}$ and inserted perpendicularly into the bilateral KI 3 (Taixi) and LR 3 (Taichong) with a depth of $20 \mathrm{~mm} .0 .25 \mathrm{~mm} \times$ $40 \mathrm{~mm}$ size needles were inserted perpendicularly into $\mathrm{CV}$ 3 (Zhongji), CV 4 (Guanyuan), the bilateral ST 25 (Tianshu), ST 28 (Shuidao), ST 29 (Guilai), ST 36 (Zusanli), and SP 6 (Sanyinjiao) with a depth of $30-35 \mathrm{~mm} .0 .30 \mathrm{~mm} \times 75 \mathrm{~mm}$ size needles were inserted obliquely into the bilateral BL 32 (Ciliao, second sacral foramina) with a depth of 50-60 mm.

For acupuncture at BL 32 (Ciliao, second sacral foramina), patients should have a strong soreness sensation which radiates to the lower abdomen. For the other points, mild reinforcing-reducing method was used, and all patients should have the "De Qi" sensation (in which patients experience a radiating feeling considered to be indicative of effective needling).

The needles were administered for a maximum of 20-30 minutes, and acupuncture treatment was administered once every other day (two prescriptions were used alternatively, 


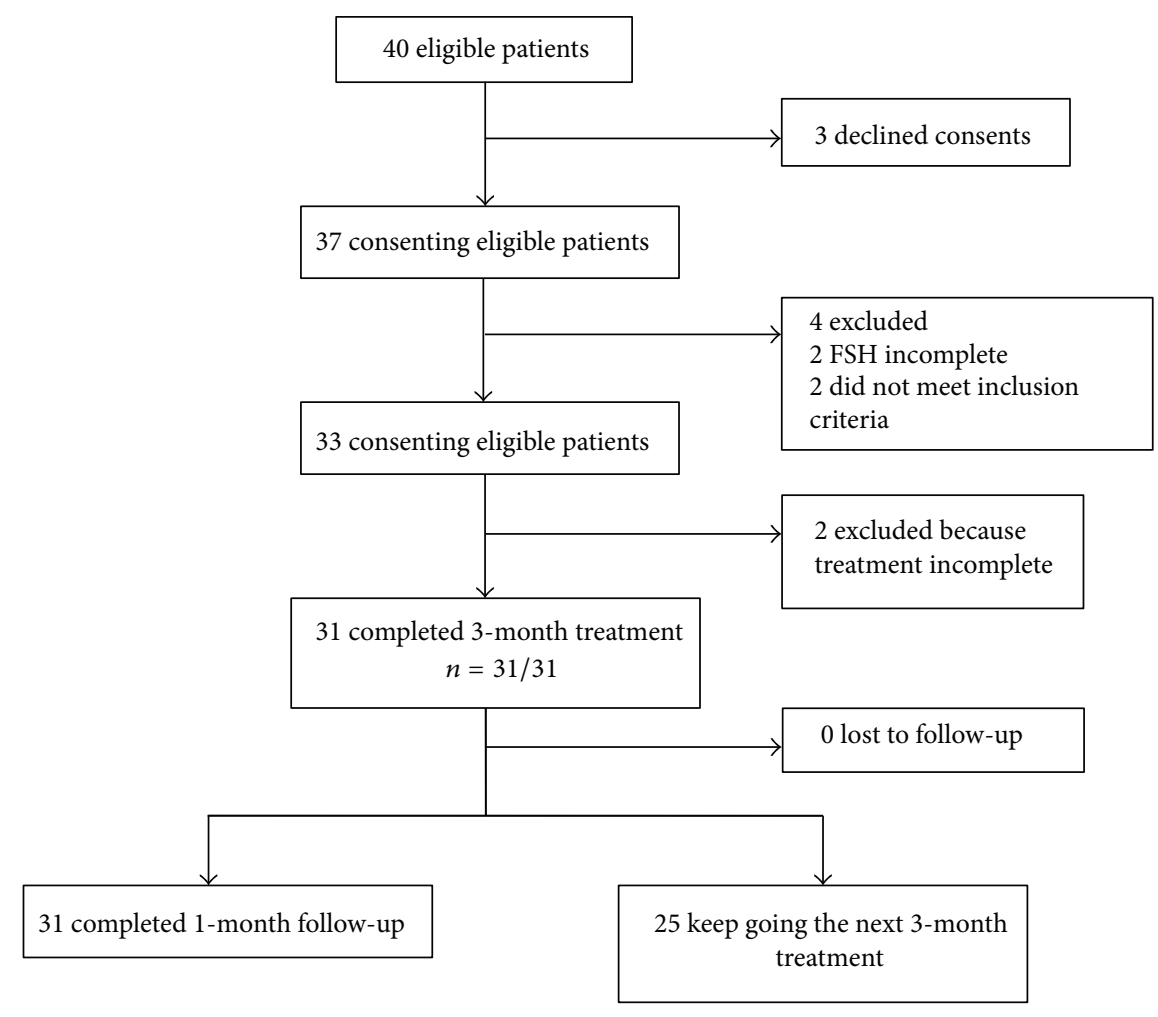

FIgURE 1: Flow chart of study.

prescription 1 twice a week and prescription 2 once a week) for three months.

2.4. Study Outcomes. The data collected including the serums $\mathrm{FSH}, \mathrm{E}_{2}$, and LH level, SAS, and Kupperman score, at baseline and after 3-month treatment. Additionally, patients' menstrual cycles were recorded according to individuals' reports during one-month follow-up. Adverse events were tracked for 3 months from initial acceptance of acupuncture treatment to the end of treatment.

2.5. Statistical Analysis. All analyses were done with SPSS software package (Version 17.0) using before and after measurements. Baseline characteristics of the patients were analyzed with conventional group descriptive statistics. $t$-test was used firstly for serums FSH, $\mathrm{E}_{2}$, and $\mathrm{LH}$ level, SAS, and Kupperman score. For the measurement data fitting normal distribution, using Two-Related-Samples Tests. If the measurement data did not fit the normal distribution, using Two-Related-Samples Tests Wilcoxon.

\section{Results}

3.1. Participant Flow. From December 2011 to May 2013, a total of 40 patients with POF visited the Hospital of Acupuncture and Moxibustion of China Academy of Chinese Medical Sciences seeking for acupuncture treatment. Of these patients, nine were excluded from the study with the following reasons: three patients declined to participate; two had incomplete FSH data; two did not meet inclusion criteria;
TABLE 2: Demographic and clinical characteristics at baseline.

\begin{tabular}{lc}
\hline Variable & $N(\%)$ or mean \pm SD \\
\hline Mean age, age & $35 \pm 4$ \\
Mean weight, kilogram & $53.7 \pm 7$ \\
Mean age at menarche, age & $14 \pm 1$ \\
History of amenorrhea, month & $8 \pm 6$ \\
History of pregnancy, $n$ & $15(48)$ \\
History of abortion, $n$ & $13(42)$ \\
History of delivery, $n$ & $5(16)$ \\
Fertility request, $n$ & $26(84)$ \\
\hline
\end{tabular}

two did not complete the whole session of treatment. (One patient moved to another city for job reason. The other one could not guarantee three times treatment per week after three-week treatment, because of her difficulties in asking for leave (Figure 1 and Table 2).)

Of these 31 patients, 25 had not been treated by acupuncture in the past, and six had been treated by acupuncture at least one month ago. And in the six, there were four received acupuncture treatment for cervical spondylosis, and two received acupuncture treatment for menstrual problem.

3.2. Baseline Characteristics. The baseline characteristics of the participants are shown in Table 2. The mean age at baseline was 35 years ( $\mathrm{SD}=4$; range, 24 40). The mean history of amenorrhea was 8 months $(S D=6$; range, 4 25). 26 of the 31 participants were with fertility request. No 


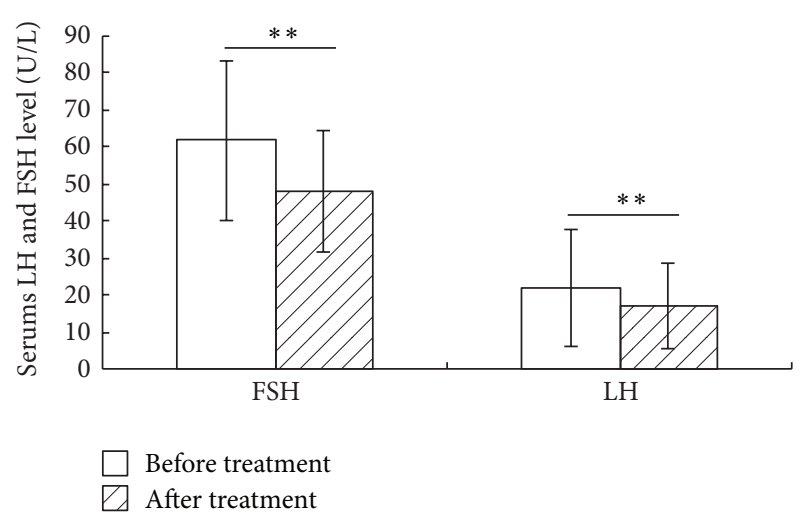

FIGURE 2: The serums LH and FSH level before and after acupuncture treatment. Both the serums FSH and LH level were reduced significantly after acupuncture treatment for 3 months $(P=0.001$, $P=0.001$, resp., $n=31)\left({ }^{* *} P<0.01\right)$.

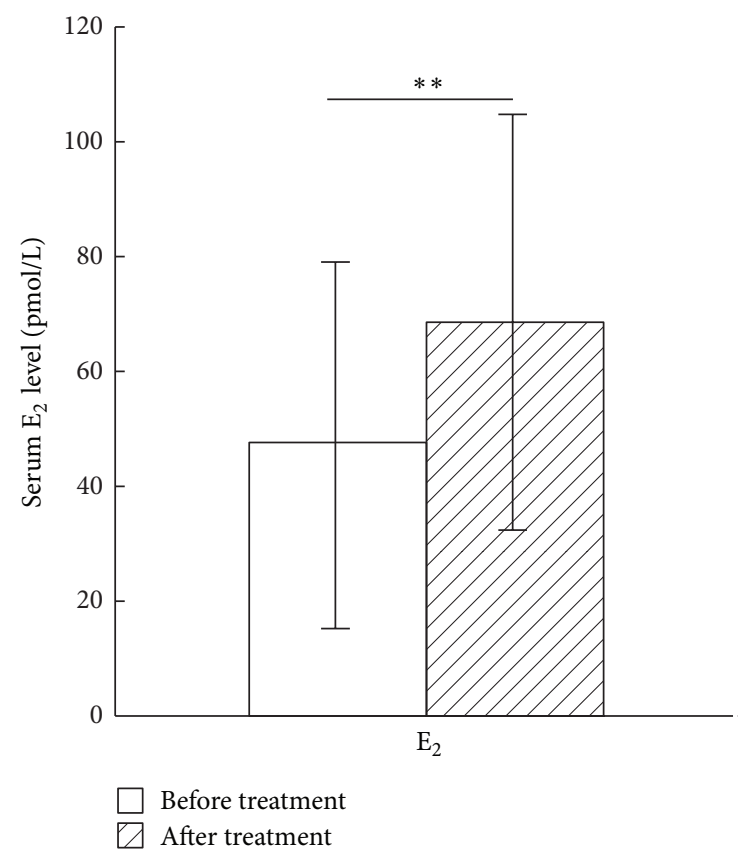

FIgURE 3: The serum $\mathrm{E}_{2}$ level before and after acupuncture treatment. The serum $\mathrm{E}_{2}$ level was reduced significantly after acupuncture treatment for 3 months $(P=0.001, n=31)\left({ }^{* *} P<0.01\right)$.

participant reported the use of hormone therapy or Chinese herbal therapy at baseline or during the entire observation period.

3.3. Effects of Acupuncture on Serums FSH and LH Level. Changes in mean serum FSH level from baseline to 3 months after final acupuncture treatment session are presented in Figure 2. Two-Related-Samples Tests Wilcoxon showed a significant reduction in the average serum FSH level at the end of treatment $(Z=4.68, P=0.001)$.

Changes in mean serum LH level from baseline to 3 months after the last time acupuncture treatment session are presented in Figure 2. Two-Related-Samples Tests showed

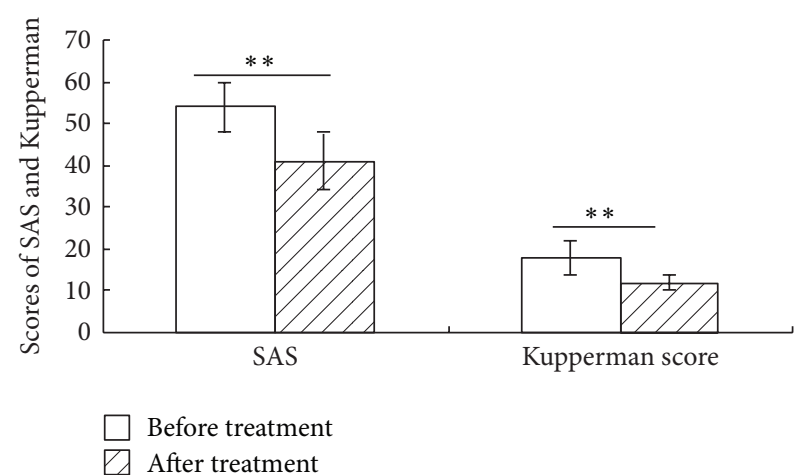

Figure 4: The scores of SAS and Kupperman before and after acupuncture treatment. Both the SAS and Kupperman scores were reduced significantly after acupuncture treatment for 3 months $(P=$ $0.000, P=0.000$, resp., $n=31)\left({ }^{* *} P<0.01\right)$.

a significant decrease in the average serum LH level at the end of treatment $(t=5.519, P=0.001)$.

3.4. Effects of Acupuncture on Serum $E_{2}$ Level. Changes in mean serum $E_{2}$ level from baseline to 3 months after final acupuncture session are presented in Figure 3. Two-RelatedSamples Tests Wilcoxon showed a significant reduction in the average serum $\mathrm{E}_{2}$ level at the end of treatment $(Z=4.48$, $P=0.001)$.

3.5. Effects of Acupuncture on the Anxiety State Assessed by SAS and the Perimenopausal Syndrome via Kupperman Score. Figure 4 showed that, during one-month baseline evaluation period, patients' SAS score was $54 \pm 6$. Two-Related-Samples Tests Wilcoxon showed a significant reduction to $41 \pm 7$ after 3-month acupuncture treatment $(Z=4.82, P=0.000)$.

Figure 4 showed that, during one-month baseline evaluation period, patients' Kupperman score was $18 \pm 4$. TwoRelated-Samples Tests Wilcoxon showed a significant reduction to $12 \pm 2$ after 3 -month acupuncture treatment $(Z=4.71$, $P=0.000)$.

3.6. Effects of Acupuncture on Menstrual Cycle. After 3-month treatment, six patients had improvement on menstruation $(6 / 31,19.4 \%)$. Four of them had menstruation after treatment, and two of them experienced menstruation during the onemonth follow-up. Of the 6 patients who regained menstruation, 2 patients regained normal color, duration, and volume of period, and 4 patients had decreased menstrual flow but with normal color and duration as compared with normal menstrual bleeding.

3.7. Safety. During the 3-month treatment, two adverse events were reported by 2 patients ( 1 small haematoma and 1 needling pain after treatment). No serious adverse events were documented.

\section{Discussion}

Although POF was not nominally recorded in TCM, its clinical manifestations can be classified into amenorrhea 
according to TCM theory. The TCM etiology of amenorrhea includes insufficient transformation of blood of the spleen and stomach, severe consumption of yin blood and exhaustion of blood source, and blockage of blood stasis in the meridians and vessels due to retention of pathogenic factors in the uterus. In this study, local and distal acupoints with functions of reinforcing liver and kidney, regulating Qi and blood, and adjusting mental activity were selected according to the amenorrhea etiology of TCM theory.

The present results indicate that the acupuncture treatment can adjust patients' serums $\mathrm{FSH}, \mathrm{E}_{2}$, and $\mathrm{LH}$ level and improve patients' SAS score and Kupperman score. The serum FSH level dropped to $48 \pm 16.6 \mathrm{IU} / \mathrm{L}$ from baseline to the end of treatment $(P=0.001)$, the serum $\mathrm{E}_{2}$ level rose to $68.24 \pm 36.15 \mathrm{pmol} / \mathrm{L}(P=0.001)$, and the serum $\mathrm{LH}$ level dropped to $17.01 \pm 11.66(P=0.001)$. The changes of hormones produced by acupuncture in present study were similar to the previous investigation using EA [24]. Modulation of serums FSH, $\mathrm{E}_{2}$, and LH level may partially explain the effects of acupuncture in treating POF, which is also observed in acupuncture improving other gynecological disorders [25].

Four patients regained menstrual cycle after treatment and two during one-month follow-up period of this study. Such result of present study is similar to the gaining of menstruation in a top athlete reported in a Japanese acupuncture study [26].

Patients' psychological comorbidities, signs, and symptoms were objectively measured with standard questionnaires by SAS and Kupperman score. The SAS score decreased to $41 \pm 7(P=0.000$ as compared with baseline), and Kupperman score decreased to $12 \pm 2(P=0.000$ as compared with baseline). The change of SAS and Kupperman score indicated that acupuncture may relieve anxiety, reduce mental stress, and improve menopausal symptoms (including hot flashes, night sweats, vaginal dryness, and mood swings).

Although POF is most frequently idiopathic or caused by autoimmune disorders, genetic causes, infections or inflammatory conditions, enzyme deficiencies, or metabolic syndromes [27], it also related to high mental and psychological distress [7]. Such cooccurrence of physical and psychological illnesses is not only associated with poor treatment response, lower quality of life, and increased healthcare costs but also related to the mutual influence of physiological processes and psychological distress that contribute to the development of POF. Present study just revealed that the SAS and Kupperman score of patients were lowered after three months' treatment. This might be explained by the mind adjustment and mental improving effects of acupuncture $[28,29]$.

Nonetheless, this present study only included 31 patients; thus the result of the study may not well characterize the general response of women with POF receiving acupuncture treatments. With an open label prospective study design and no control group, researcher could not eliminate these confounding factors. Of the 31 patients, there were two patients had acupuncture treatment in other hospitals 1.5 and 2 months before participating in our study. Because the detailed information of their previous acupuncture treatment (like acupoints selected and stimulating methods applied) was not fully recorded, we could not compare those two acupuncture regimens. This is a methodological drawback of present study. Thus, the detailed relevant information about previous treatment of participants should be fully and meticulously recorded in future study. Meanwhile, in order to increase the objectivity and fairness of observational study, inclusion and exclusion criteria of future study should be stricter. Excluding those patients who have had other treatments may reduce confounding factors.

Commonly used acupuncture formulas for amenorrhea in China normally include acupoints with functions of reinforcing liver and kidney and regulating Qi and blood. Compared to the commonly used formulas, our acupuncture regimen added acupoints for adjusting mental activity. This may partially explain the different therapeutic effects between the two patients' previous formula and ours.

Although the significant changes of serums $\mathrm{FSH}, \mathrm{E}_{2}$, and LH level in this study were most likely due to acupuncture effects, those serum hormones' levels did still not reach the normal level after treatment. To test the therapeutic effectiveness of acupuncture, further randomized control trials are needed.

\section{Conclusion}

The present results showed the feasibility and safety of acupuncture for the treatment of POF in China. These findings suggest that acupuncture may decrease serums FSH and $\mathrm{LH}$ level, raise serum $\mathrm{E}_{2}$ level, relieve anxiety, reduce mental stress, and improve the menopausal symptoms. No serious side effects were found.

\section{Conflict of Interests}

The authors declare that no competing financial interests exit.

\section{Authors' Contribution}

Yigong Fang conducted acupuncture treatment for all patients and had overall responsibility for the trial. Jinsheng Yang contributed to the study design. Yingru Chen collected the data and wrote the report, which was reviewed by all authors. Fei Wang collected data. Yingying Wang and Li Yang did the literature search for this study.

\section{Acknowledgments}

This study was supported by Independent Project of Acupuncture Research Institute of China Academy of Chinese Medical Sciences (Project no. ZZ07001). The authors thank the patients who participated in the trial and Acupuncture Hospital of China Academy of Chinese Medical Sciences for providing lunch during research.

\section{References}

[1] R. W. Rebar, "Premature ovarian failure," Obstetrics and Gynecology, vol. 113, no. 6, pp. 1355-1363, 2009. 
[2] L. M. Nelson, "Primary ovarian insufficiency," The New England Journal of Medicine, vol. 360, pp. 606-614, 2009.

[3] C. K. Welt, "Primary ovarian insufficiency: a more accurate term for premature ovarian failure," Clinical Endocrinology, vol. 68, no. 4, pp. 499-509, 2008.

[4] P. A. H. van Noord, J. S. Dubas, M. Dorland, E. Te Velde, and H. Boersma, "Age at natural menopause in a population-based screening cohort: the role of menarche, fecundity, and lifestyle factors," Fertility and Sterility, vol. 68, no. 1, pp. 95-102, 1997.

[5] L. T. Shuster, D. J. Rhodes, B. S. Gostout, B. R. Grossardt, and W. A. Rocca, "Premature menopause or early menopause: longterm health consequences," Maturitas, vol. 65, no. 2, pp. 161-166, 2010.

[6] J. C. Gallagher, "Effect of early menopause on bone mineral density and fractures," Menopause, vol. 14, no. 3, part 2, pp. 567571, 2007.

[7] J. G. van der Stege, H. Groen, S. J. N. van Zadelhoff et al., "Decreased androgen concentrations and diminished general and sexual well-being in women with premature ovarian failure," Menopause, vol. 15, no. 1, pp. 23-31, 2008.

[8] J. Zhou and F. Qu, "Treating gynaecological disorders with traditional Chinese medicine: a review," African Journal of Traditional, Complementary and Alternative Medicines, vol. 6, no. 4, pp. 494-517, 2009.

[9] F. Qu, J. Zhou, X. Sang, X. Wang, and R. Nan, "Acupuncture and auricular acupressure in relieving menopausal hot flashes of bilaterally ovariectomized Chinese women: a randomized controlled trial," Evidence-Based Complementary and Alternative Medicine, vol. 2011, Article ID 713274, 8 pages, 2011.

[10] D. I. Kim, J. C. Jeong, K. H. Kim et al., "Acupuncture for hot flushes in perimenopausal and postmenopausal women: a randomised, shamcontrolled trial," Acupuncture in Medicine, vol. 29, no. 4, pp. 249-256, 2011.

[11] A. Vincent, D. L. Barton, J. N. Mandrekar et al., "Acupuncture for hot flashes: a randomized, sham-controlled clinical study," Menopause, vol. 14, no. 1, pp. 45-52, 2007.

[12] K. H. Kim, K. W. Kang, D. I. Kim et al., "Effects of acupuncture on hot flashes in perimenopausal and postmenopausal womena multicenter randomized clinical trial," Menopause, vol. 17, no. 2, pp. 269-280, 2010.

[13] H. Jin, T.-T. Liu, and R. Wang, "Clinical observation on acupuncture at the five-zangshu for treatment of perimenopausal syndrome," Zhongguo Zhen Jiu, vol. 27, no. 8, pp. 572-574, 2007.

[14] C. H. Zheng, M. M. Zhang, G. Y. Huang et al., "The role of acupuncture in assisted reproductive technology," EvidenceBased Complementary Alternative Medicine, vol. 2012, Article ID 543924, 15 pages, 2012.

[15] C. H. Zheng, G. Y. Huang, M. M. Zhang, and W. Wang, "Effects of acupuncture on pregnancy rates in women undergoing in vitro fertilization: a systematic review and meta-analysis," Fertility and Sterility, vol. 97, no. 3, pp. 599-611, 2012.

[16] W. W. Zung, "A rating instrument for anxiety disorders," Psychosomatics, vol. 12, no. 6, pp. 371-379, 1971.

[17] H. P. G. Schneider, L. A. J. Heinemann, H.-P. Rosemeier, P. Potthoff, and H. M. Behre, "The menopause rating scale (MRS): comparison with Kupperman index and quality-of-life scale SF36," Climacteric, vol. 3, no. 1, pp. 50-58, 2000.

[18] Z. Y. Wang and Y. F. Chi, "Self-rating anxiety scale," Shanghai Archives of Psychiatry, no. 2, pp. 73-74, 1984.
[19] X.-Y. Wang, H.-Y. Yang, G.-N. Nie et al., "Study on the reliability and validity of the Chinese Menopause Rating Scale (CMRS)," Chinese Journal of Epidemiology, vol. 29, no. 9, pp. 882-886, 2008.

[20] L. M. Nelson, J. N. Anasti, M. R. Flack et al., "Premature ovarian failure," in Reproductive Endocrinology, Surgery, and Technology, E. Y. Adashi, J. A. Rock, and Z. Rosenwaks, Eds., pp. 1393-1410, Lippincott-Raven, Philadelphia, Pa, USA, 1996.

[21] R. W. Rebar and H. V. Connolly, "Clinical features of young women with hypergonadotropic amenorrhea," Fertility and Sterility, vol. 53, no. 5, pp. 804-810, 1990.

[22] G. S. Conway, "Premature ovarian failure," British Medical Bulletin, vol. 56, no. 3, pp. 643-649, 2000.

[23] WHO Regional Office for the Western Pacific, WHO Standardized Acupuncture Point Location in the Western Pacific, World Health Organization, Manila, Philippines, 2008.

[24] K. H. Zhou, J. G. Jiang, J. N. Wu et al., "Electroacupuncture modulates reproductive hormone level in patients with primary ovarian insufficiency: results from a prospective observationl study," Evidence-Based Complementary and Alternative Medicine, vol. 2013, Article ID 657234, 7 pages, 2013.

[25] J. Johansson, L. Redman, P. P. Veldhuis et al., "Acupuncture for ovulation induction in polycystic ovary syndrome: a randomized controlled trial," American Journal of PhysiologyEndocrinology and Metabolism, vol. 304, no. 9, pp. E934-E943, 2013.

[26] N. Donoyama, S. Hotoge, and N. Ohkoshi, "Acupuncture might have contributed to improving amenorrhoea in a top athlete," Acupuncture in Medicine, vol. 29, no. 4, pp. 304-306, 2011.

[27] N. Santoro, "Mechanisms of premature ovarian failure," Annales d'Endocrinologie, vol. 64, no. 2, pp. 87-92, 2003.

[28] W. D. Wang, X. Y. Lu, S. M. Ng et al., "Effects of electroacupuncture on personality traits in depression: a randomized controlled study," Chinese Journal of Integrative Medicine, vol. 19, no. 10, pp. 777-782, 2013.

[29] F. Carvalho, K. Weires, M. Ebling et al., "Effects of acupuncture on the symptoms of anxiety and depression caused by premenstrual dysphoric disorder," Acupuncture in Medicine, vol. 31, no. 4, pp. 358-363, 2013. 


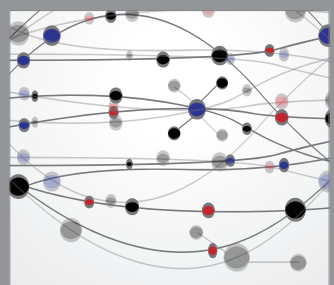

The Scientific World Journal
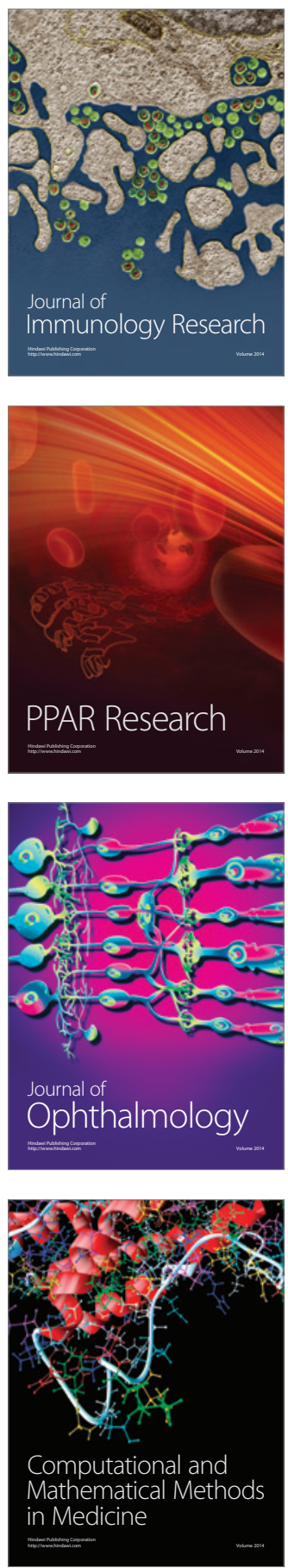

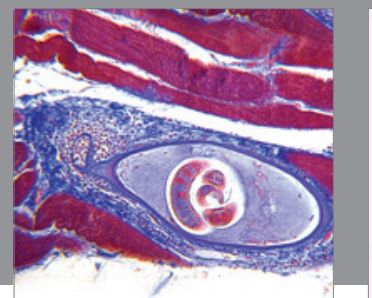

Gastroenterology

Research and Practice
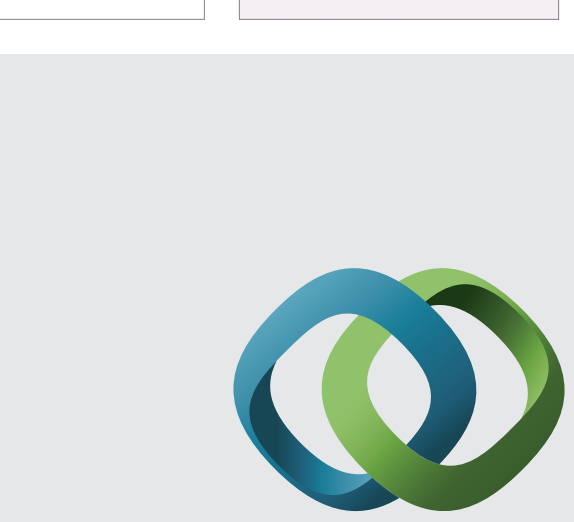

\section{Hindawi}

Submit your manuscripts at

http://www.hindawi.com
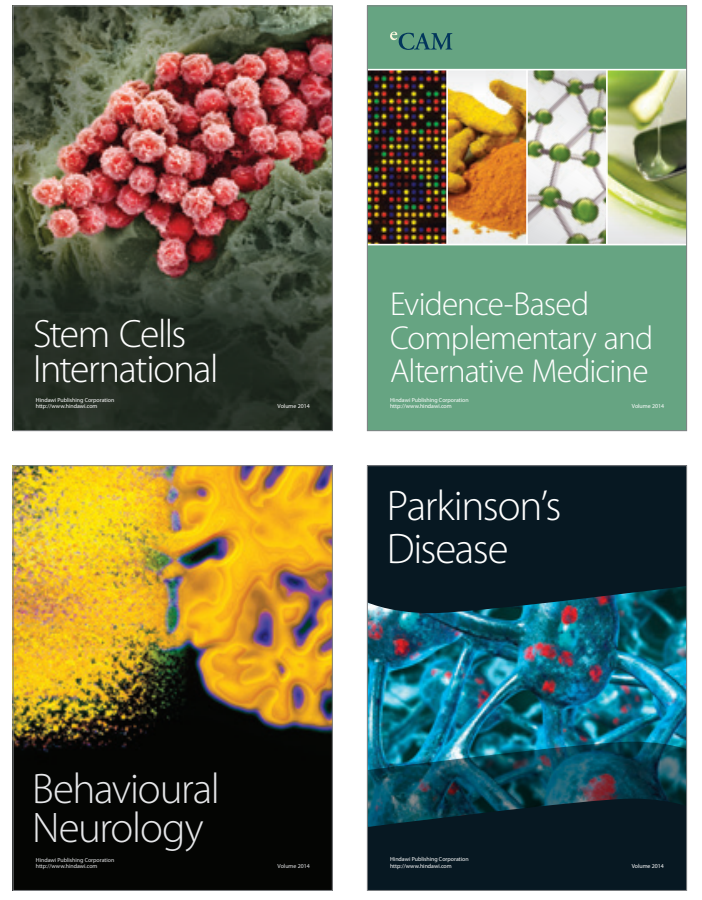
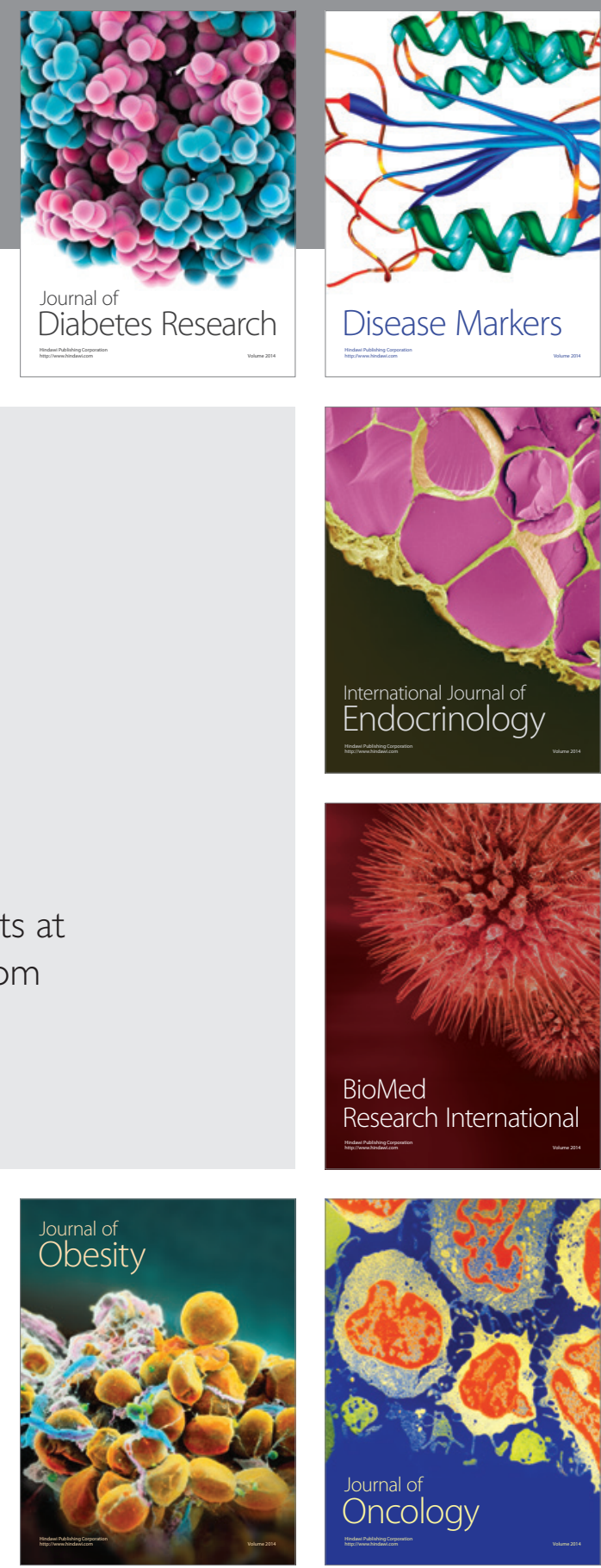

Disease Markers
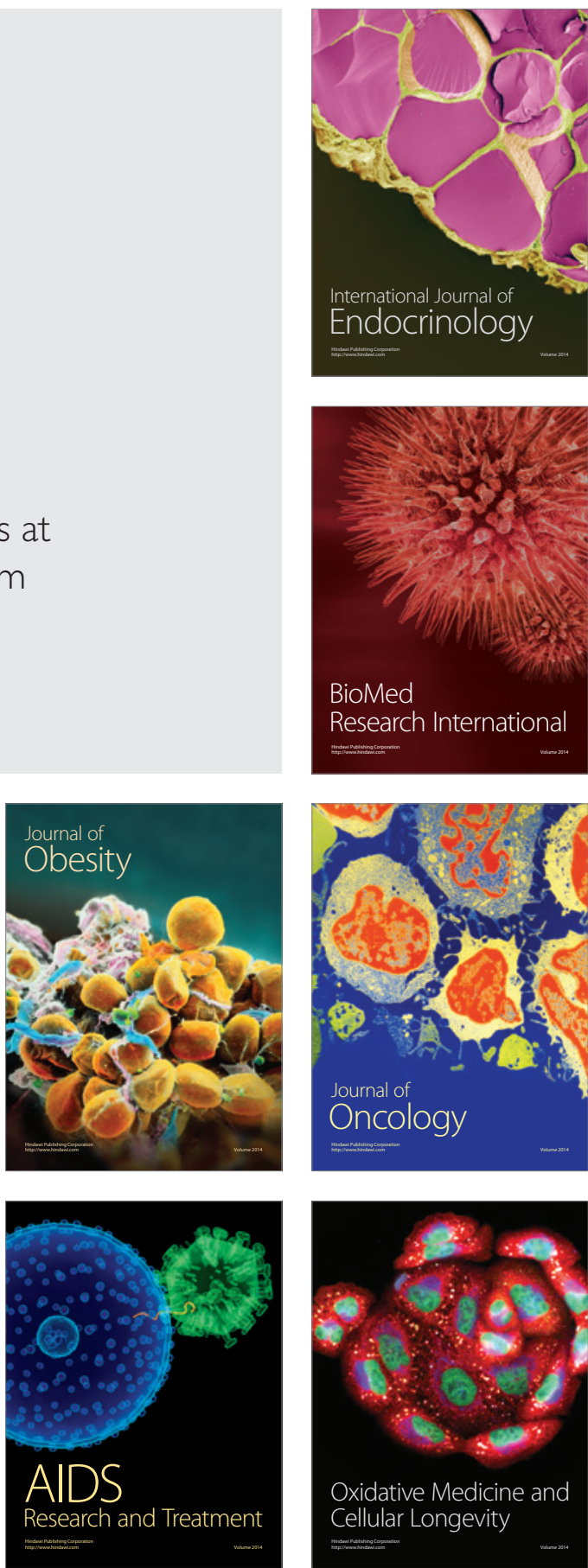